\title{
Optical-induced absorption tunability of Barium Strontium
}

\section{Titanate film}

\author{
Chunya Luo ${ }^{1,4}$, Jie $\mathrm{Ji}^{1}$, Jin Yue ${ }^{1}$, Yunkun $\mathrm{Rao}^{2}$, Gang $\mathrm{Yao}^{1}$, Dan $\mathrm{Li}^{4}$, Ying Zeng ${ }^{2}$, Renkui \\ $\mathrm{Li}^{2}$,Longsheng Xiao ${ }^{4}$, Xinxing Liu ${ }^{1}$, Jianquan Yao ${ }^{1,3,4}$, Furi Ling ${ }^{* 2}$ \\ ${ }^{\text {I}}$ Wuhan National Laboratory for Optoelectronics, Huazhong University of Science and Technology, Wuhan \\ 430074, China \\ ${ }^{2}$ School of Optical and Electronic Information, Huazhong University of Science and Technology, \\ Wuhan 430074, China \\ ${ }^{3}$ College of Precision Instrument and Opto-electronics Engineering, Tianjin University, Tianjin 300072, \\ China \\ ${ }^{4}$ Institute of Information Science and Technology, Department of Physics and Mechanical \& Electrical \\ Engineering, Hubei University of Education, Wuhan 430205, China \\ Corresponding author,e-mail: lingfuri@mail.hust.edu.cn
}

\begin{abstract}
The absorption tunability of $100 \mathrm{~nm}$ thickness of ferroelectric Barium Strontium Titanate $\left(\mathrm{Ba}_{0.5} \mathrm{Sr}_{0.5} \mathrm{TiO}_{3}\right)$ thin films with different densities of pumped optical field is measured by terahertz time-domain spectroscopy in the range of $0.2 \mathrm{THz}-1.2 \mathrm{THz}$ at $19^{\circ} \mathrm{C}$. Experimental results show that the absorption coefficient of BST film is approximately at 5000 $\mathrm{cm}^{-1}-20000 \mathrm{~cm}^{-1}$ in the range of $0.2 \mathrm{THz}-1.2 \mathrm{THz}$ and the absorption coefficient reached up to $16 \%$ when we applied the optical field up to $600 \mathrm{~mW}$. The theoretical calculations reveal that increasing photoexcitation fluences is responsible for the increasingof transmission change in the conduction current density cause the absorption coefficient varied.
\end{abstract}

Keywords: optical tunability; absorption response; $\mathrm{BaSrTiO}_{3} ; \mathrm{THz}$

\section{Introduction}

For the sake of comprehensive application prospects in various areas such as optical, electric, and microwave devices in wireless communications and control systems, and possessing the excellent nonlinear optical properties, ferroelectric and electro-optical physical properties, ferroelectric materials have drew great attention both in scientific research and technology development [1]. In which, the ferroelectric materials Barium Strontium Titanate $\left(\mathrm{Ba}_{x} \mathrm{Sr}_{1-\mathrm{x}} \mathrm{TiO}_{3}\right.$, abbreviated as BST) has been researched for several decades. BST materials has widely used in the microwave tunable devices and dynamic random access memories [2,3]. BST materials are promising candidates for use in the design of electronic devices intended for controlling radiation in the optical and microwave frequency ranges [4]. $\mathrm{Ba}_{1-\mathrm{x}} \mathrm{Sr}_{\mathrm{x}} \mathrm{TiO}_{3}$ is currently a material for microwave applications due to its low loss and composition dependent Curie temperature (Tc) [5], which ranges from -163 to $120^{\circ} \mathrm{C}$ for $\mathrm{x}=0$ to $\mathrm{x}=1$ respectively. We chose that the $\mathrm{Ba} / \mathrm{Sr}$ ratio is 1 to realize better tunability, for in that condition, the $\mathrm{Tc}$ is just at around room temperature, which is more favorable for practical device application. In order to make up the defects of the bulk BST, such as the sintering temperature demands very high to obtain good crystallinity, the dielectric constant is too high to inevitably leads to a very complicated impedance 
matching circuit [5], we prefer to do the research on the BST film.

Thin film BST offers advantages over bulk BST for tunable applications. Large electric fields $(0-400 \mathrm{kV} / \mathrm{cm})$ can be achieved in thin film BST $(224 \mathrm{~nm})$ using low voltages [5]. And on the modulate properties of the thin film BST, many methods have been proposed in literature. The agility of the modulate properties comes from the modification of temperature [6] or external dc (low-frequency) electric field [7]. The generalization of terahertz (THz) communicating systems in the objects of space program and military contributes significantly to the development of miniature and agile modulators. The use of a single reconfigurable modulator allows tuning its operating frequency to different communications standards and so, permits replacing multiband elements in standard systems. Ferroelectric materials, which are characterized by a high permittivity up to the THz range [7] offer a possibility of its tuning by means of external field. Whereas, these tunable properties are most researched in the microwave region $[8,9]$ or in the terahertz range but by the means of dc electric-field [7-9], or by the means of codoping and adjusting sintering temperature for attain the high modulation of $\mathrm{Ba} 0.67 \mathrm{Sr}_{0.33} \mathrm{TiO}_{3}$ ceramics [5], or adopted the method of temperature control [5]. One of the works where STO shows large change in the complex refrtactive index with temperature [10], although the modulation capability is superior to the pumped optical tunability, the thermal tunability was not so accurate. Due to the excellent optical performance, many properties of the film have been experimentally and theoretically studied [11].

In his work, the transmission spectrum of BST film in the THz range with a varied external pumped optical field is measured. The absorption coefficient of the BST thin film at THz frequencies was derived from the measurement data. The pumped optical field dependence of absorption coefficient of the BST thin film thus evaluated at THz frequencies.

\section{Experiments}

The BST film samples prepared via a standard magnetron sputtering method. The films were sputtered from a stoichiometric $\left(\mathrm{Ba}_{0.5} \mathrm{Sr}_{0.5}\right) \mathrm{TiO}_{3}$ target onto $\mathrm{TiO}_{2}(100 \mathrm{~nm}) / \mathrm{SiO}_{2}(100 \mathrm{~nm}) / \mathrm{Si}$ substrates. And then the step profiler reveals a thickness of $104 \mathrm{~nm}$ ( $4 \%$ accuracy). The dimensions of the studied substrates were $2 \mathrm{~mm} \times 2 \mathrm{~mm} \times 0.5 \mathrm{~mm}$.

The THz time domain spectrum (THz-TDS) system produced by the Zomega Terahertz Corporation of the US was used to measure the transmittance spectrum at $19^{\circ} \mathrm{C}$, which the signal to noise ratio of the terahertz data/measurements was above the $50 \mathrm{~dB}$. Figure 1 shows that in the fiber femtosecond laser beam was divided into two beams (named the pump light and the probe beam, respectively) by a polarized beam splitter. The $\mathrm{THz}$ pulses transmitted through the sample were detected using a usual electro-optic sampling scheme with another $1 \mathrm{~mm}$ thick ZnTe crystal and a pair of balanced Si photodiodes. The TDS probes the in-plane response of the sample. The measured frequency resolution is $10 \mathrm{GHz}$, and the spot size is $3 \mathrm{~mm}$ in diameter. An all-solid-state continue-wave (cw) green laser (center wavelength $532 \mathrm{~nm}$ ) was employed in the experiment to provide external optical pumping. The light is obliquely incident upon the surfaces of the samples at an angle of $45^{\circ}$ and the spot size is $5 \mathrm{~mm}$ in diameter [12]. To make sure that the sample restored to its previous dynamic behavior, we performed a thermal process after each measurement. The sample was placed in a furnace at $220^{\circ} \mathrm{C}$ for $3 \mathrm{~h}$, and no external field was applied to it in this recovery process. 


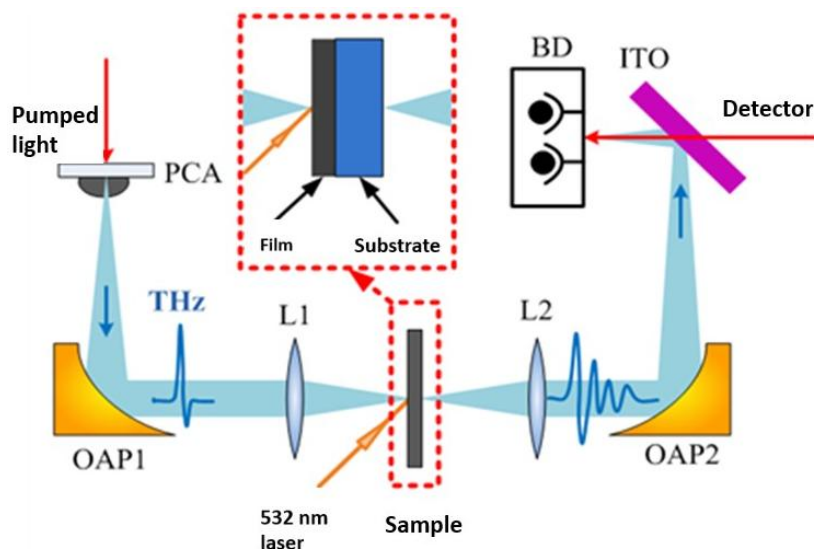

Fig. 1. (color online) Installation diagram of the TDS. A green laser is obliquely incident upon the surface of the sample at an angle of $45^{\circ}$ with respect to the polar axis.

In the experiments the sample was casted with the different external optical densities fields onto the BST film. A $532 \mathrm{~nm}$ green laser was employed in the experiment to provide external optical pumping and the optical density was increased to $600 \mathrm{~mW}$. We calculated the complex transmittances and the intrinsic phases in frequency domain from the waveforms in Fig. $2 b$ through a Fourier transform. The experiments was carried out in two steps [7]: firstly, measurement of a signal wave form $E_{s}$ with the thin film on a substrate in the path of the THz beam; and secondly, measurement of a reference wave form Eref with a bare substrate see Fig. 2a.

\section{Results and discussion}
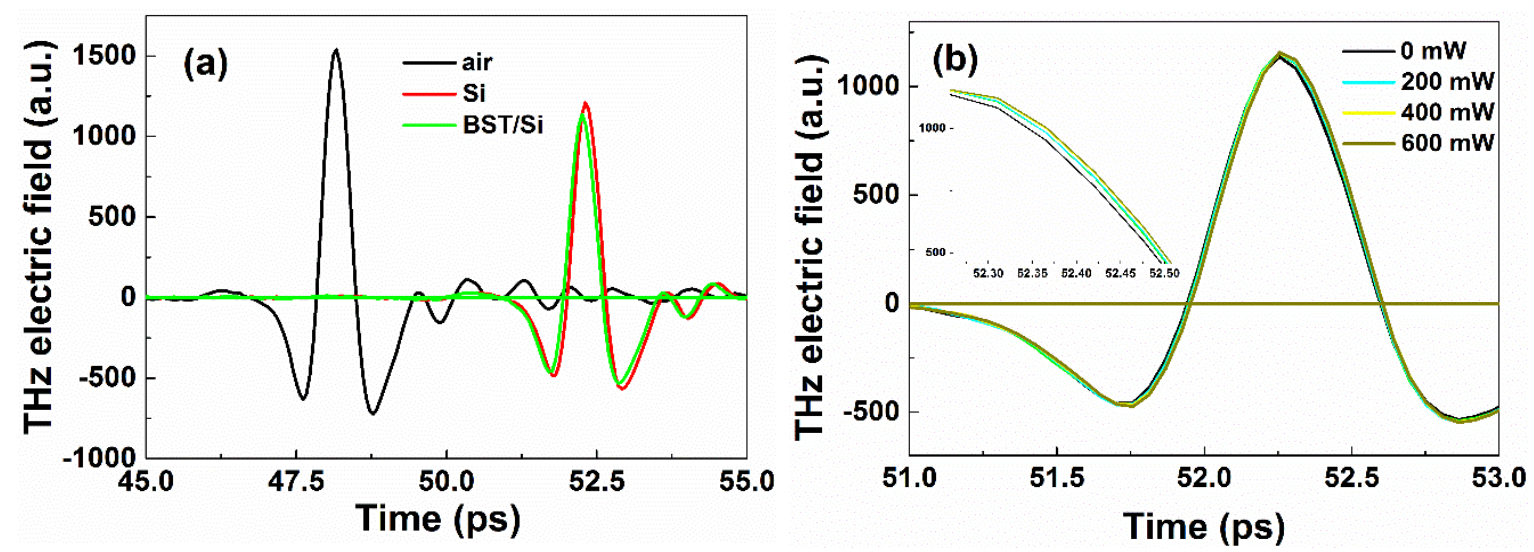

Fig. 2. (color online) THz wave forms: (a) sample without external optical field at $19^{\circ} \mathrm{C}$; the data serve for the evaluation of the thin film properties without optical field and (b) sample with external optical field; $0 \mathrm{~mW}$ is a reference wave form without pumped optical field. (Inset) Comparison of the THz electric field variation with different pumped optical fields.

Figure 2a demonstrates the reference wave form Eref with the substrate and Fig.2b shows the signal results of the zero-field $(0 \mathrm{~mW})$ wave form and the difference wave form of 200, 400 and 600 $\mathrm{mW}$ optical density applied. As can be seen that these signals almost have the same shape except little change with increasing optical field amplitude, as shows in Fig.2a that the transmittance waveform shifts about 0.025 ps when the pumped optical field power is $600 \mathrm{~mW}$, compared to the without pumped optical field, and that the transmitted $\mathrm{THz}$ signal increases when the pumped optical field increasing. 
We calculate the transmission spectrum and the intrinsic phase shift of the sample by the Fourier-transform method: $T(\omega)=\frac{E_{S}(\omega)}{E_{r e f}(\omega)}$.

We have calculated the complex refractive index spectrum of the BST film in the research. Taking account of the multiple reflections inside the Si substrate, both the real and imaginary parts of the refraction index of the BST thin film have been evaluated from the measured waveforms using Fresnel's equations [13].The technique allows us to determine the refractive index of the thin film in the range from $0.2 \mathrm{THz}$ to $1.2 \mathrm{THz}[14]: \quad N_{f}(\omega)=n(\omega)+i k(\omega)$. In which, the $n(\omega)$ and the $k(\omega)$ are the real and imaginary parts of the refractive index of the film, respectively. The complex refractive index $\mathrm{N}_{\mathrm{f}}$ was then retrieved by numerically inverting the expression for $T(\omega)[14]$, from the $\mathrm{THz}$ transmittance spectra by formula (1).

$$
T(\omega)=\frac{2 N_{f}\left(N_{s}+1\right) \exp \left[i \omega\left(N_{f}-1\right) d_{f} / c\right]}{\left(N_{f}+1\right)\left(N_{f}+N_{s}\right)+\left(1-N_{f}\right)\left(N_{f}-N_{s}\right) \exp \left[i \omega\left(N_{f} d_{f} / c\right)\right]}
$$

Here, $d_{f}$ is the film thickness, c is the speed of light and $N_{s}$ is the complex optical refractive index of the substrate as a function of $\omega$.And in order to investigate the influence of the pumped optical field on the refractive index of BST film, we calculate the frequency dependence of the real and imaginary part of the complex refractive index as shown in Fig. 3a and 3b. Fig.3 demonstrates the optical properties of the BST film at the different external optical fields. The detectable frequency range is from $0.2 \mathrm{THz}$ to $1.2 \mathrm{THz}$. The value of the real part of the refraction index is $35 \sim 50$, and the imaginary part of the refraction index 10 40. It is showed that the $\mathrm{n}$ demonstrated a decreasing with the optical density increased and the k showed a inversely trendency, which shows a certain tunability of the optical property.
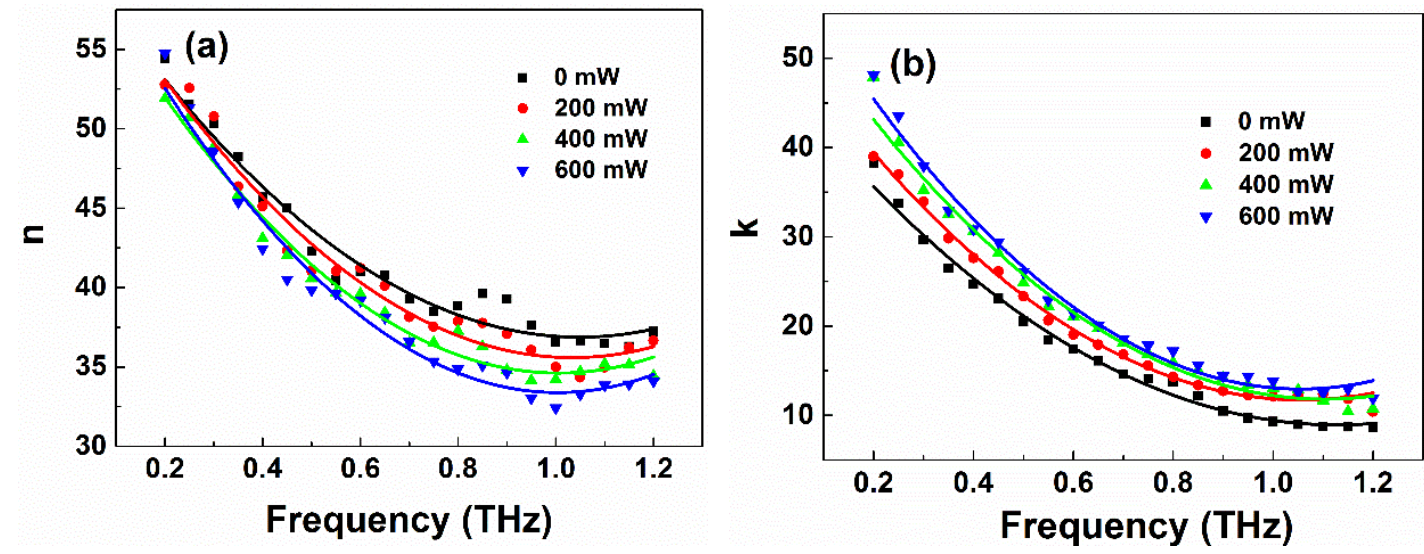

Fig. 3. (color online) Frequency dependences of optical properties (a) real parts of the refraction index and (b) imaginary parts of the refraction index for the BST film with different densities of pumped optical field at $19^{\circ} \mathrm{C}$.

In order to investigate the absorption properties of the BST film, the frequency dependence of absorption coefficient was calculated with the Eq.(2), as shown in Fig. 4a. 


$$
k(\omega)=\alpha(\omega) c / 2 \omega
$$

As can be seen from the Fig. $4 \mathrm{a}$ it is found that the absorption coefficient is about 15000 in the range and the absorption coefficient is decreasing with the frequency increased. For evaluate the optical-induced absorption coefficient properties of the thin film, we use the following formula: $\frac{\Delta \alpha}{\alpha}=\frac{|\alpha(I)-\alpha(0)|}{\alpha(0)} \times 100 \%$, and Fig. $4 \mathrm{~b}$ shows the absorption variation rate of the BST film with different pumped optical fields. It is demonstrated that the absorption coefficient modulation show a certain behavior upon the pumped optical field. The absorbance coefficient is increased with the external pumped optical density increasing. And the variation absorption rate shows a linear relationship with the pumped density. And the results of Fig. 4b show that the optical-field induced absorption coefficient change is found to be tunable by up to $16 \%$ via the application of an external optical field of $600 \mathrm{~mW}$ when the frequency is $0.98 \mathrm{THz}$. Though the modulation capacility of the superconductor[16,17] and the active graphene-silicon hybrid film[18] are supurior to the ferroelectric BST film, the modulator demands the samples prepared with metamaterial, they demands a more complicated samples.
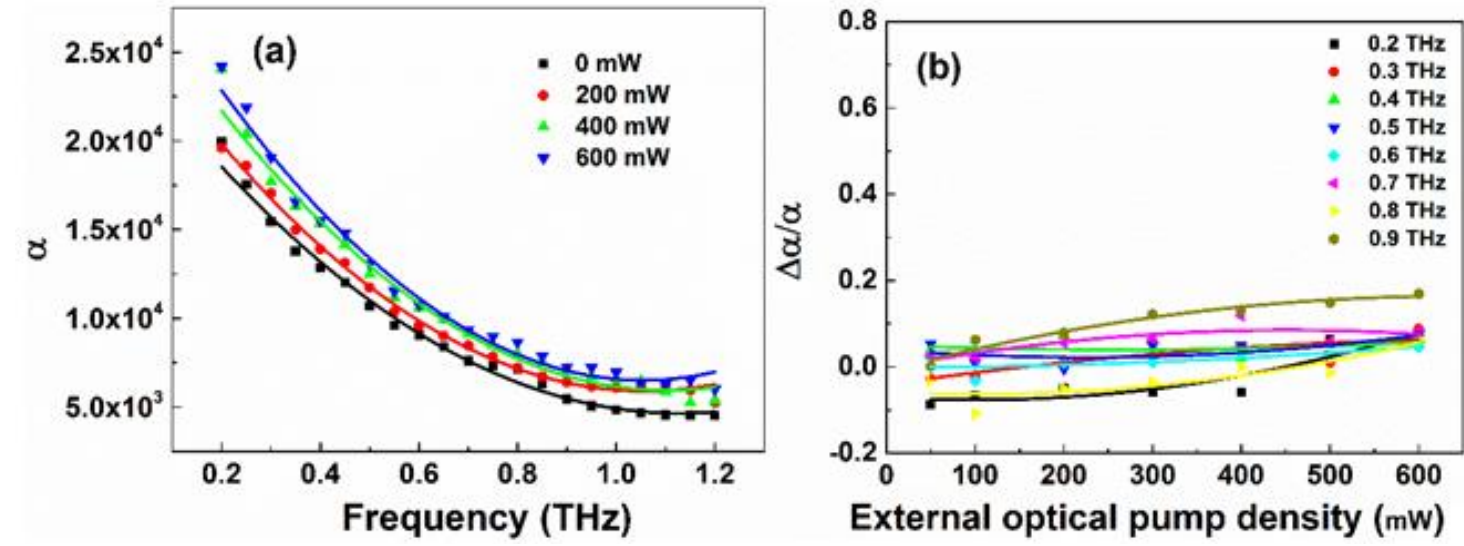

Fig. 4. (color online) Frequency dependences of (a) absorption coefficient and (b) tunability of absorption coefficient for BST film with different densities of pumped optical field at $19^{\circ} \mathrm{C}$.

Here the mechanism behind the observed absorption characteristics can be explained with respected to the conduction loss which determined by the pumped optical field. For the BST film, the applied external optical field would lead to the Ti ions and the imputies for absorbing the pumped optical energy. The process would lead to a redistribution of Ti ions and the imputies, which special displacement of the carries leads to a the internal space charge field and corresponding variation of

refractive index via a linear electro-optic effect. i.e.

$$
\Delta n=\frac{1}{2}\left(n_{o}^{3} \gamma_{13}-n_{e}^{3} \gamma_{33}\right) \propto E \propto I, \text { where }
$$

$n_{\mathrm{o}}, n_{\mathrm{e}}, \gamma_{i j}, E$ and $I$ are refractive index of ordinary and extrraordinary light, linear electro-optic coefficient, internal space charge field along the polar axis and applied light density as shown in Fig.5, 
due to the drift and diffusion of the electrons, the conduction current density would be developed in this process. This result indicates that the change of the absorption coefficient of the film may be attributed to the change of conduction current density resulted from the internal space charge field in the sample caused by the pumped optical field.

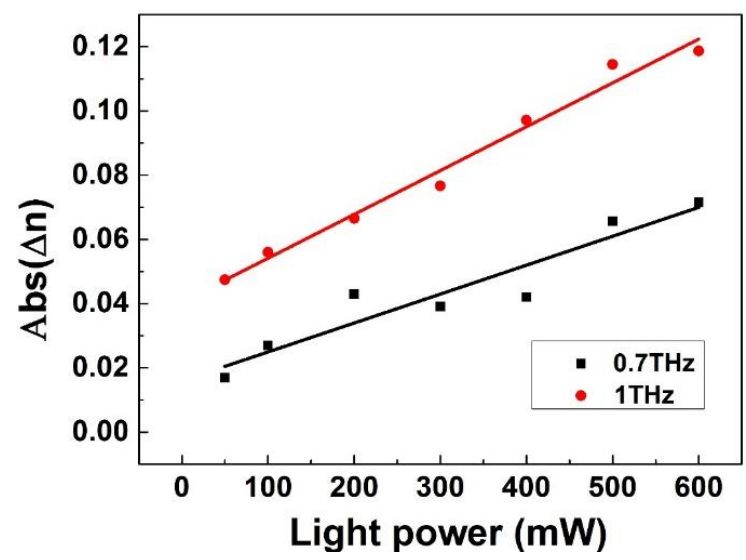

FIG.5. Light intensity dependence of variation of refraction index of BST film at 0.7 and $1 \mathrm{THz}$. The dots are experimental value and the solid line are the fitting.

\section{Conclusion}

To summarize, the optical field-induced tunable the absorption coefficient properties of the BST film in the $\mathrm{THz}$ frequency range are experimentally and theoretically discussed. The absorbance coefficient of thin film irradiated by optical fields exhibit tunability up to $16 \%$ when the pumped opticalfild attained $600 \mathrm{~mW}$, this variation of the absorption coefficient are attribute to the conduction current density and we verified the analysis by the experiment result. This provide the distinct possibility applications for tunable device applications in multifunctional responses.

\section{Acknowledgments:}

This work was supported by the National Basic Research Program (973 Program, Grant No.2015CB755403) and the CAEP THz Science and Technology Foundation (No.AEPTHZ201407) and the National Science Foundation of Hubei Province (No. 2014CFB562 and No. 2015CFB502) and the project of Hubei Ministry of Education (No.Q20152904 and No. B2015011).

\section{References}

[1] M. Liu, C. Ma, G. Collins, J. Liu, C. Chen, A.D. Alemayehu, G. Subramanyam, Y. Ding, J. Chen, C. Dai, Nanoscale research letters, 8 (2013), pp. 1-6.

[2] A. Chanthbouala, V. Garcia, R.O. Cherifi, K. Bouzehouane, S. Fusil, X. Moya, S. Xavier, H. Yamada, C. Deranlot, N.D. Mathur, M. Bibes, A. Barthelemy, J. Grollier, Nat Mater, 11 (2012), pp. 860-864.

[3] S. Li, Y. Yao, Y.I. Yuzyuk, Y. Jia, X. Wang, S. Xie, Y. Jing, Applied Physics Letters, 105 (2014), p. 092901.

[4] G.A. Komandin, A.A. Volkov, I.E. Spektor, K.A. Vorotilov, V.M. Mukhortov, Physics of the Solid State, 51 (2009), pp. 1351-1355.

[5] D. Yan, L. Luo, Y. Zhang, Z. Peng, H. Liu, D. Xiao, T. Liu, X. Lai, J. Zhu, Ceramics International, 41 (2015), pp. S520-S525.

[6] D. Fuchs, C.W. Schneider, R. Schneider, H. Rietschel, Journal of Applied Physics, 85 (1999), p. 7362. 
[7] P. Kužel, F. Kadlec, H. Němec, R. Ott, E. Hollmann, N. Klein, Applied Physics Letters, 88 (2006), p. 102901.

[8] Y.L. Cheng, Y. Wang, H.L.W. Chan, Integrated Ferroelectrics, 70 (2005), pp. 151-157.

[9] V.K. Palukuru, J. Peräntie, J. Jäntti, H. Jantunen, International Journal of Applied Ceramic Technology, 9 (2012), pp. 11-17.

[10] R. Singh, A. K. Azad,Q. X. Jia,A. J. Taylor, .H.-T. Chen,Optics Letters,36(2011), pp.1230-1232

[11] K.X. Jin, B. Yang, Y. Zhang, B.C. Luo, L.Y. Chen, C.L. Chen, Scripta Materialia, 112 (2016), pp. 62-66.

[12] L. Wu, L. Jiang, Y. Xu, X. Ding, J. Yao, Applied Physics Letters, 103 (2013), p. 191111.

[13] N. Kida, M. Hangyo, M. Tonouchi, Physical Review B, 62 (2000), p. R11965.

[14] P. Kužel, F. Kadlec, Comptes Rendus Physique, 9 (2008), pp. 197-214.

[15] L. Wu, F.-R. Ling, Z.-G. Zuo, J.-S. Liu, J.-Q. Yao, Chinese Physics B, 21 (2012), p. 017802.

[16] R.Singh, J.Xiong, A. K. Azad, H.Yang, S. A.Trugman, Q. X.Jia, A.J.Taylor, H.-T.Chen, Nanophotonics, 1 (2012), pp. 117 - 123

[17] R. Singh, D. R. Chowdhury, J. Xiong, H. Yang, A. K. Azad, A. J. Taylor, Q. X. Jia, H.-T. Chen, Applied Physics Letters, 103 (2013), p.061117.

[18]Q. Li, Z. Tian, X.-Q. Zhang, R. Singh, L.-L. Du, J.-Q. Gu, J.G. Han, W.I. Zhang, Naure Communications, 6 (2015), p.7082. 